\title{
Performance of an automated human immunodeficiency virus (HIV) antigen/antibody combined assay for prenatal screening for HIV infection in pregnant women
}

Fourth-generation antigen/antibody $(\mathrm{Ag} / \mathrm{Ab})$ combined assays, which allow the simultaneous detection of anti-human immunodeficiency virus (anti-HIV) antibodies and HIV p24 core protein in serum or plasma, are increasingly used for both the diagnosis of and screening for HIV infection. These assays have been shown to reduce the diagnostic window by a mean of 4 or 5 days compared with third-generation HIV-Ab detection assays (Weber et al., 1998). Nevertheless, discrepant results have been published on their specificities in population groups with low prevalence of HIV infection, such as blood donors (Bourlet et al., 2005; Gürtler et al., 1998; Kwon et al., 2006; Ly et al., 2000, 2004, 2007; Sickinger et al., 2004; Weber et al., 1998, 2002). Prenatal screening for HIV infection is currently recommended to prevent vertical HIV transmission. Pregnancy has been recognized as a risk factor for false-positive results in HIV immunoassays (Celum et al., 1994; Doran \& Parra, 2000; Magee et al., 1999; Zacharias et al., 2004). In the present study we determined the rate of falsepositive results with a fourth-generation HIV Ag/Ab combined assay used for the routine prenatal screening for HIV infection and compared it to that of a third-generation HIV immunoassay.

Until 1 March 2005, prenatal screening for HIV infection was carried out with a thirdgeneration Ab assay (AxSYM HIV 1/2/gO test; Abbott Laboratories). Reactive samples [signal to cut-off ratio (S/CO) $\geqslant 1.0$ ] were reassayed after centrifugation. A second serum specimen from patients testing repeatedly positive was requested for further analysis, which included testing by the same immunoassay, and, for reactive samples, analysis by a line immunoassay (LIA) (INNO-LIA HIV I/II Score; Innogenetics). Since 1 March 2005, a fourth-generation immunoassay (Architect HIV Ag/Ab Combo; Abbott
Laboratories) has been used at our laboratory, for both diagnosis and screening purposes. Reactive specimens $(\mathrm{S} / \mathrm{CO} \geqslant 1)$ were centrifuged and reanalysed in duplicate following the manufacturer's instructions. A second serum specimen and a plasma sample from patients displaying a repeatedly reactive test result in the HIV combined test were immediately requested for additional testing, which included confirmation of serum reactivity by the same HIV combined assay, and LIA testing for reactive specimens, and analysis of plasma HIV RNA load (Cobas Amplicor HIV-1 Monitor version 1.5 or Cobas Ampliprep/Cobas TaqMan HIV-1; both from Roche Diagnostics Systems). Repeatedly reactive samples were considered false-positive results if reactivity was absent on repeat sampling, and they tested negative by the LIA (until March 2005), or by the LIA and the plasma HIV RNA assay (from March 2005). A number of serum specimens were tested by an HIV p24 Ag-detection assay (Innotest HIV 1/2 Ag MAB; Innogenetics).

Relevant data from this study are shown in Table 1. From January 2002 to March 2005, 7026 blood sera specimens (from 6971 patients) were received at our laboratory for prenatal screening for HIV infection. Overall, five sera $(0.07 \%)$ from three patients $(0.04 \%)$ tested repeatedly reactive (weakly positive, $\mathrm{S} / \mathrm{CO}<3$ ) in the third-generation immunoassay but negative in the LIA, and were thus considered false-positive results. Over this period of time, the prevalence of HIV infection in this population group was $0.22 \%$. The per-sample and per-patient positive predictive values (true positives/ true positives + false positives) of the assay were 86.1 and $84.2 \%$, respectively. From March 2005 to May 2008 (an equivalent period of time), 12063 blood sera samples (from 11989 patients) were analysed. A total of 49 sera $(0.40 \%)$ from 27 patients $(0.22 \%)$ tested repeatedly reactive (all samples but one - which had a S/ $\mathrm{CO}=17.3$ - displaying $\mathrm{S} / \mathrm{COs}<3$ ) in the fourth-generation immunoassay, but negative by LIA. Plasma samples $(n=27)$ from these patients tested negative (undetectable viral load) in the plasma HIV RNA assay. Over this period of time, the prevalence of HIV infection was $0.16 \%$. The per-sample and per-patient positive predictive values of the assay were 44.3 and $57.4 \%$, respectively.

The sera giving false-positive results in the third-generation HIV Ab assay $(n=5)$ were retrieved and analysed by the HIV Ag/Ab combined assay. All these sera tested repeatedly positive (weakly reactive, S/CO $<3)$. Likewise, available sera $(n=20)$ of those yielding false-positive results in the HIV combined test were analysed by the AxSYM HIV 1/2/gO assay, and with an HIV p24-Ag-detection assay. Two sera tested positive in the former assay (S/CO 1.2 and 1.7). All sera tested negative in the latter test.

HIV Ag/Ab combined immunoassays may potentially yield a higher rate of falsepositive results than third-generation $\mathrm{Ab}$ assays, as the former combine two reactions of a different nature, which may require different cut-off thresholds for optimal specificity and sensitivity, in a single test. Data published a few years ago appeared to support this assumption (Gürtler et al., 1998; Ly et al., 2000; Weber et al., 1998). Nevertheless, the specificity of recently marketed HIV combined assays has been reported to be comparable to that of third-generation immunoassays (Bourlet et al., 2005; Kwon et al., 2006; Ly et al., 2004, 2007; Sickinger et al., 2004; Weber et al., 2002). Pregnancy has been recognized as a risk factor for false-positive results in HIV immunoassays (Celum et al., 1994; Doran \& Parra, 2000; Magee et 
Table 1. Data on the performance of a third-generation HIV immunoassay and a fourth-generation HIV Ag/Ab combined assay in the routine prenatal screening for HIV infection

\begin{tabular}{|lcccc|}
\hline \multirow{2}{*}{ Assay } & Period of time & \multicolumn{2}{c|}{ Result (no. of samples/no. of patients) } \\
\cline { 3 - 5 } & & True positive & False positive & Negative \\
\hline AXSYM HIV 1/2/gO & January 2002-March 2005 & $31 / 16$ & $5 / 3$ & $6990 / 6952$ \\
Architect HIV Ag/Ab Combo & March 2005-May 2008 & $39 / 20$ & $49 / 27$ & $11975 / 11942$ \\
\hline
\end{tabular}

al., 1999; Zacharias et al., 2004). Our data suggest that the Architect HIV Ag/Ab Combo test yields a higher rate of falsepositive results than the third-generation AxSYM HIV 1/2/gO immunoassay for pregnant women undergoing prenatal screening for HIV infection, a population group with a low prevalence of HIV infection. Whether or not this difference was statistically significant could not be appropriately addressed, as sera were not run in parallel for both tests. Further studies are thus needed to clarify this. In a comparable study (Fitzpatrick \& Kaminski, 2006), the AxSYM HIV Ag/Ab combined assay (equivalent to the Architect assay) was also shown to yield a higher rate of false-positive results than the AxSYM HIV 1/2/gO test. The cause of false-positive reactivities in the Architect HIV combined test is unclear. In most cases, however, a spurious reaction between the patients' sera with the test Ags could be ruled out, as the sera gave negative results in the thirdgeneration immunoassay, which employs a similar combination of HIV recombinant Ags and synthetic peptides. Most likely, a non-specific binding of an unknown serum component with the $\mathrm{p} 24$-specific $\mathrm{mAb}$ may account for the false-positive reactivities. The extent could not be confirmed though, as none of the sera were reactive in a p24 Ag-detection assay. Nevertheless, the epitope specificity of the anti-p24 mAbs in both assays may be different. Although most false-positive results in the HIV combined test gave $\mathrm{S} / \mathrm{COs}<3$, and thus we were suspicious of them being false-positive results, the performance of complementary tests is required to rule out a current $\mathrm{HIV}$ infection. The need for additional testing results in the deferral of the delivery of the report, which may create uncertainty and anxiety for the woman and her family.

\section{Tomás García, ${ }^{1}$ Nuria Tormo, ${ }^{1}$ Concepción Gimeno, ${ }^{1,2}$ Juan García de Lomas $^{1,2}$ and David Navarro ${ }^{1,2}$}

\section{${ }^{1}$ Microbiology Service, Hospital Clínico Universitario, Valencia, Spain}

${ }^{2}$ Department of Microbiology, School of Medicine, University of Valencia, Valencia, Spain

\section{Correspondence: David Navarro (david.navarro@uv.es)}

Bourlet, T., Pretis, C., Pillet, S., Lesenechal, M., Piche, J. \& Possetto, B. (2005). Comparative evaluation of the VIDAS HIV DUO ultra assay for combined detection of HIV-1 antigen and antibodies to HIV. J Virol Methods 127, 165-167.

Celum, C. L., Coombs, R. W., Jones, M., Murphy, V., Fisher, L., Grant, C., Corey, L., Inui, T., Wener, M. H. \& Holmes, K. K. (1994). Risk factors for repeatedly reactive HIV-1 EIA and indeterminate Western blots. A populationbased case-control study. Arch Intern Med 154, 1129-1137.

Doran, T. I. \& Parra, E. (2000). False-positive and indeterminate human immunodeficiency virus test results in pregnant women. Arch Fam Med 9, 924-929.

Fitzpatrick, F. \& Kaminski, G. (2006). Use of a fourth-generation HIV assay for routine screening - the first year's experience. J Infect 53, 415-416.

Gürtler, L., Mühlbacher, A., Michl, U., Hofmann, H., Paggi, G. G., Bossi, V., Thorstensson, R., G-Villaescusa, R., Eiras, A. \& other authors (1998). Reduction of the diagnostic window with a new combined p24 antigen and human immunodeficiency virus antibody screening assay. J Virol Methods 75, 27-38.

Kwon, J. A., Yoon, S. Y., Lee, C. K., Lim, C. S., Lee, K. N., Sung, H. J., Brennan, C. A. \& Devare, S. G. (2006). Performance evaluation of three automated human immunodeficiency virus antigen-antibody combination immunoassays. J Virol Methods 133, 20-26.
Ly, T. D., Edlinger, C., Vabret, A., Morgan, O. \& Greuet, B. (2000). Contribution of combined detection assays of p24 antigen and antihuman immunodeficiency virus (HIV) antibodies in diagnosis of primary HIV infection by routine testing. J Clin Microbiol 38, 24592461.

Ly, T. D., Laperche, S., Brennan, C., Vallari, A., Ebel, A., Hunt, J., Martin, L., Daghfal, D., Schochetman, G. \& Devare, S. (2004). Evaluation of the sensitivity and specificity of six HIV combined p24 antigen and antibody assays. J Virol Methods 122, 185-194.

Ly, T. D., Ebel, A., Faucher, V., Fihman, V. \& Laperche, S. (2007). Could the new HIV combined antigen and antibody assay replace p24 antigen specific assay? J Virol Methods 143, 86-94.

Magee, L. A., Murphy, K. E. \& Von Dadelszen, P. (1999). False-positive results in antenatal HIV screening. CMAJ 160, 1285.

Sickinger, E., Stieler, M., Kaufman, B., Kapprell, H. P., West, D., Sandrige, A., Devare, S., Schochetman, G., Hunt, J. C. \& other authors (2004). Multicenter evaluation of a new, automated enzyme-linked immunoassay for detection of human immunodeficiency virusspecific antibodies and antigen. J Clin Microbiol 42, 21-29.

Weber, B., Fall, E. M. B., Berger, A. \& Doerr, H. W. (1998). Reduction of diagnostic window by new fourth-generation human immunodeficiency virus screening assays. J Clin Microbiol 36, 2235-2239.

Weber, B., Gürtler, L., Thostensson, R., Michl, U., Mühlbacher, A., Bürgisser, P., Villaescusa, R., Eiras, A., Gabriel, C. \& other authors (2002). Multicenter evaluation of a new automated fourth-generation human immunodeficiency virus screening assay with a sensitive antigen detection module and high specificity. J Clin Microbiol 40, 1938-1946.

Zacharias, N. M., Athanassaki, I. D., Sangi-Haghpeykar, H. \& Gardner, M. O. (2004). High false-positive rate of human immunodeficiency virus rapid serum screening in a predominantly Hispanic prenatal population. J Perinatol 24, 743-747. 